\title{
Redescription d'Heligmosomoides travassosi Schulz, 1926 (Nématode Héligmosominé), parasite de Cricetus cricetus L. (Cricétidé)
}

\author{
par Marie-Claude DURETTE-DESSET
}

Laboratoire de Zoologie (Vers), associé au C.N.R.S. (Pr A.-G. ChaBaud), Muséum national d'Histoire naturelle, 57, rue Cuvier, F 75005 Paris

\section{Résumé}

Heligmosomoides travassosi Schulz, 1926, parasite de Cricetus cricetus, originaire de Strasbourg (France) est redécrit.

Cette espèce présente de grandes variations morphologiques des côtes 9-10-11 chez le ớ. Les spermatozoïdes sont remarquables par leur grande taille. De plus, alors que chez le $\delta$ il sont unis deux à deux et de forme allongée, dans l'utérus de la $q$, ils prennent une forme globuleuse.

\section{Summary}

Redescription of Heligmosomoides travassosi Schulz, 1926 (Nematoda Heligmosominae) parasite of Cricetus cricetus L. (Cricetidae).

The ribs 9-10-11 show important morphological variations in the $\delta$ of this species. The spermatozoa are characterized by their large size. Moreover they are found in pairs and have an elongated shape in the $\delta$, whereas they have a globulous shape in the uterus of the ?. 


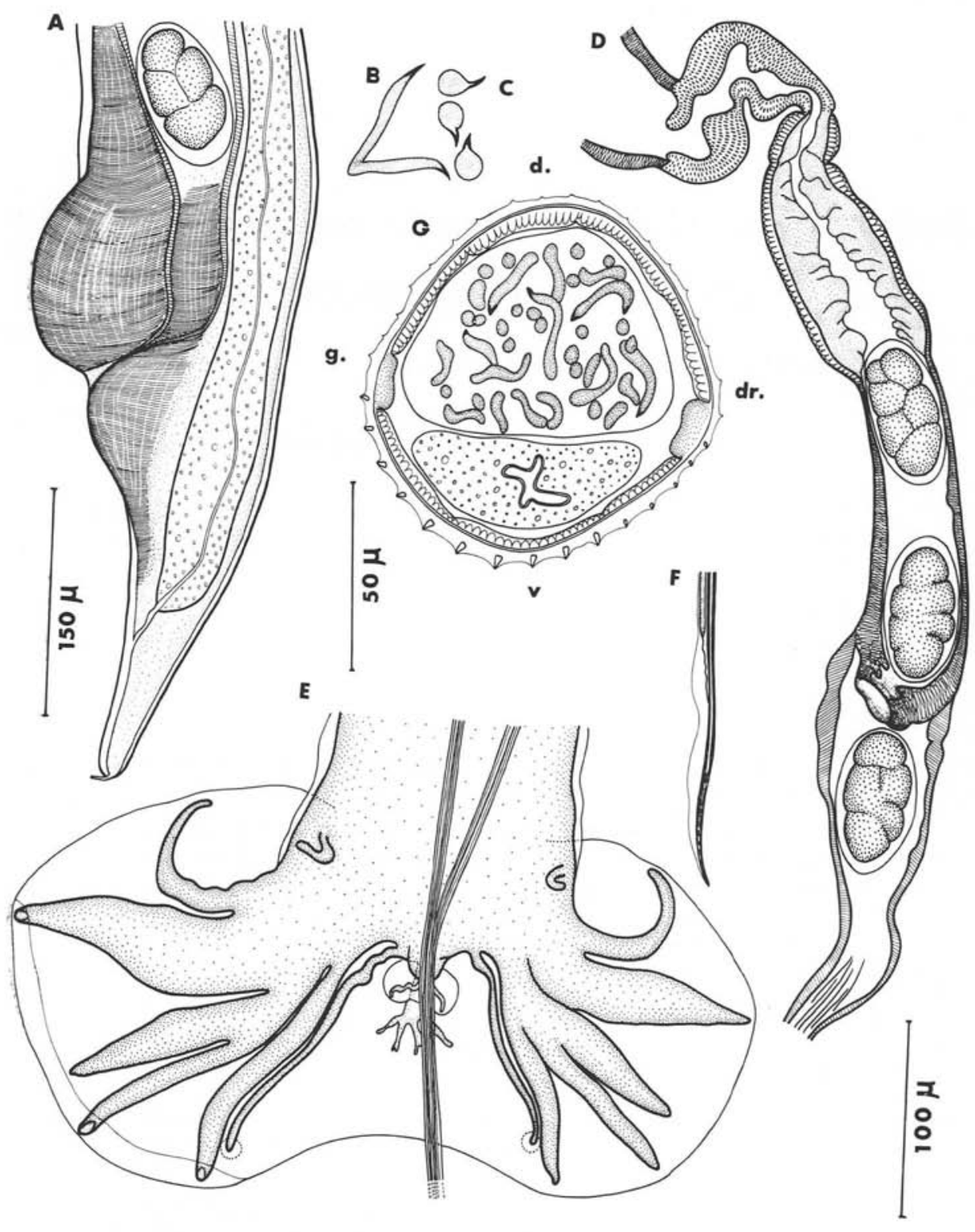

Fig. 1. - Heligmosomoides travassosi Schulz, 1926. A, $q$, extrémité postérieure, vue latérale

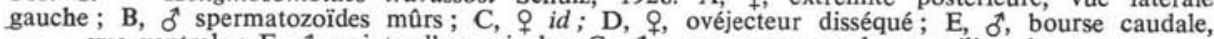
vue ventrale; F, $\delta$, pointe d'un spicule; $\mathrm{G}$, $\delta$, coupe transversale au milieu du corps. A, D, éch. : $150 \mu$ E, éch. : $100_{\mu}, \mathrm{B}, \mathrm{C}, \mathrm{F}, \mathrm{G}$, éch. : $50_{\mu}$ 
Comme nous l'avons noté dans un précédent travail (cf. Durette-Desset, 1967): le genre Heligmosomoides paraît être inféodé aux Microtidés et les espèces les plus primitives se trouvent dans un foyer centre-européen. Cependant, et presque uniquement en Europe, quelques espèces ont évolué chez des hôtes de capture. C'est le cas d'Heligmosomoides travassosi Schulz, 1926, parasite de Cricetus cricetus et dont des spécimens nous ont été envoyés à deux reprises de la région de Strasbourg (France) par MM. F. Rodhain et F. Petter que nous remercions vivement. Nous remercions également M. le professeur Callot qui nous a fait parvenir un spécimen monté entre lame et lamelle.

Il nous a paru utile de redécrire cette espèce, car chez le $\delta^{*}$, il existe de grandes variations morphologiques bursales (voir fig. 2). D'autre part, nous avons pu observer un changement de forme des spermatozoïdes chez le $\sigma^{*}$ (fig. 1, B) et dans l'utérus de la $q$ (fig. 1, C). Enfin, la $q$ présente une formation particulière, au niveau de la vulve, située entre la cuticule et les muscles (fig. 1, E et fig. 3).

\section{Matériel étudié :}

- $15 \stackrel{9}{\uparrow} 12 \delta^{*}$, parasites d'un Cricetus cricetus L. originaire de Strasbourg (France); tube 735 SA.

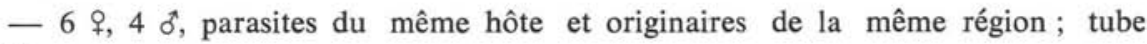
$871 \mathrm{M}$.

Ces spécimens sont déposés dans les collections du Muséum National d'Histoire Naturelle de Paris.

\section{Description.}

Nématodes de petite taille présentant, surtout chez les $q$, un enroulement sénestre très serré le long de la ligne ventrale. Les tours de spires sont d'environ $4 \mathrm{chez}$ le $\delta$ et $6 \mathrm{chez}$ la 9 . Chez les deux sexes, la partie postérieure du corps est déroulée.

Vésicule céphalique haute de $55 \mu$ sur $38 \mu$ de large. Position du pore excréteur variable. Deirides filiformes.

\section{SYNLOPHE.}

Chez les deux sexes, le corps est parcouru longitudinalement dans sa partie moyenne par 34 crêtes cuticulaires chez le ô, $43 \mathrm{chez}$ la $\uparrow$. Comme dans les autres espèces du genre, les crêtes ventrales sont les plus marquées et l'orientation se fait de la droite vers la gauche (fig. 1, G). Chez le $\sigma^{\star}$ les crêtes disparaissent à environ $80 \mu$ en avant de la bourse caudale, chez la $\uparrow$, les crêtes dorsales disparaissent les premières, puis les ventrales, au niveau de la trompe. 


\section{MÂLE :}

Corps long de $5,7 \mathrm{~mm}$ sur $130 \mu$ de large dans sa partie moyenne. Anneau nerveux, pore excréteur et deirides situés respectivement à $250 \mu, 500 \mu$ et $450 \mu$ de l'apex. Esophage long de $510 \mu$. Le testicule débute à $300 \mu$ en arrière de l'œsophage.
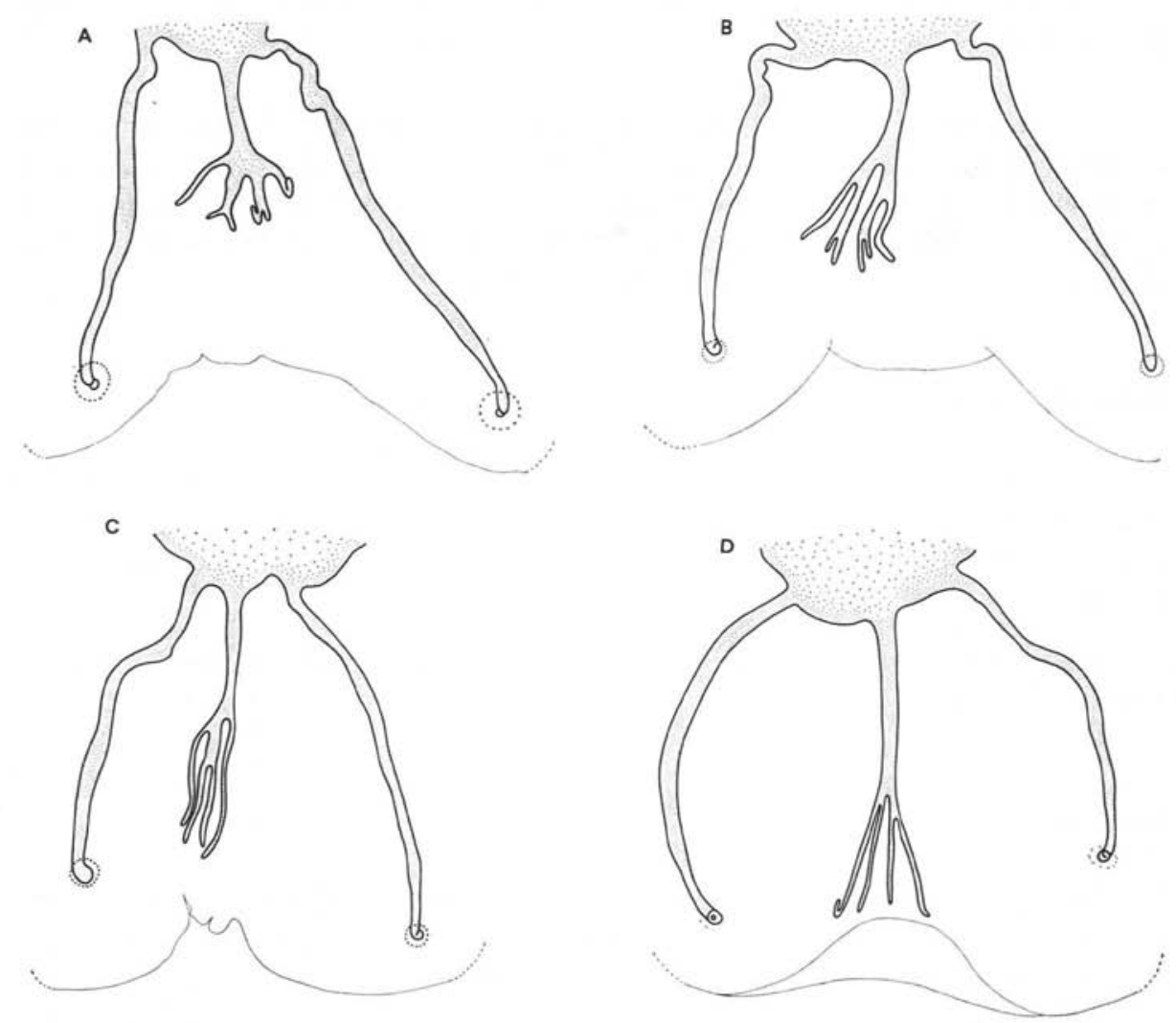

FIG. 2. - Heligmosomoides travassosi Schulz, 1926. A, B, C, D. $\sigma^{7}-$ variations des côtes 8, 9 et 10.

Bourse caudale légèrement asymétrique avec un lobe droit plus développé, haute de $155 \mu$ sur $385 \mu$ de large.

Côtes externo-dorsales naissant à la racine de la côte dorsale, légèrement inégales, la droite étant la plus longue. Elles présentent un renflement interne situé près de leur racine. Côte dorsale courte, de $52 \mu$ de long, divisée à peu près à la moitié de sa hauteur en 4 rameaux sensiblement égaux. Les rameaux internes sont bifurqués à leur extrémité (fig. 1, E). Cône génital portant 2 grandes expansions foliacées sur sa lèvre postérieure (fig. 1, E). 
Spicules sub-égaux, longs de $620 \mu$, dédoublés sauf à leur extrémité et se terminant chacun par une longue pointe de $25 \mu$ (fig. 1, F). Dans le canal déférent, les spermatozoïdes mûrs se présentent unis deux à deux sous la forme d'un tube long de $60 \mu$ sur $4 \mu$ de large (fig. 1, B). Les deux extrémités pointues constituent les noyaux (colorés en rouge par le Feulgen).

Il est remarquable de constater que dans l'utérus de la $q$, les spermatozoïdes, qui n'ont alors qu'un seul noyau, prennent une forme ovoïde (fig. 1, C) ; un phénomène comparable a déjà été mis en évidence par Foor W.E., Johnson M. H. et Beaver P. C., 1971, chez Dipetalonema viteae.

\section{VARIATIONS.}

Chez le Cricetus cricetus 735 SA, nous avons observé des variations assez importantes chez les $12 \delta$ étudiés, ces variations portant essentiellement sur les caractères de la bourse caudale. En considérant uniquement la longueur de la côte dorsale, le niveau de la naissance des côtes 9 et la fusion des côtes 10 et 11 , les spécimens peuvent être répartis en 3 types :

- type $a$ : côte dorsale courte, côtes 9 naissant à peu près au même niveau que les côtes 10 . Côtes 10 et 11 non fusionnées. C'est le type qui correspond au travassosi décrit par Schulz (fig. 2, A);

- type $b$ : côte dorsale longue, côtes 9 naissant après la séparation des côtes 10 . Côtes 10 et 11 non fusionnées (fig. 2, B);

- type $c$ : côte dorsale presque aussi longue que les côtes 8 , côtes 9 naissant avant la séparation des côtes 10 . Côtes 10 et 11 fusionnées (fig. 2, C).

Les variations peuvent également porter sur la présence ou l'absence d'un renflement interne à la racine des côtes 8 .

Nous n'avons pas pu mettre en évidence de corrélation entre ces différents caractères; d'autre part, certains spécimens possèdent des caractères intermédiaires entre les trois types (par exemple, côte dorsale très longue et 10 et 11 fusionnées comme dans le type $c$, mais côtes 9 naissant presque au niveau des côtes 10 comme dans le type $a$ (fig. 2, D). Nous pensons donc qu'il s'agit dans tous les cas de la même espèce.

\section{Femelle.}

Corps long de $7,4 \mathrm{~mm}$, large de $150 \mu$ dans sa partie moyenne. Vésicule céphalique haute de $90 \mu$ sur $40 \mu$ de large. Anneau nerveux, pore excréteur et deirides situés respectivement à $220 \mu, 400 \mu$ et $400 \mu$ de l'apex. CEsophage long de $590 \mu$.

Appareil génital monodelphe. La vulve s'ouvre à $185 \mu$ de la pointe caudale. Vagin long de $60 \mu$. Comme chez tous les Heligmosomoides, le vestibule est très allongé $(610 \mu)$; seule sa partie proximale est muscularisée, la partie médiane, de longueur sensiblement égale, se termine en faisant saillie dans la partie proximale (voir fig. 1, D). Sphincter et trompe respectivement longs de $32 \mu$ et $140 \mu$. L'utérus long de 1,6 mm contenait 22 œufs non embryonnés (fig. 1, D). 
Queue longue de $110 \mu$ avec une pointe de $15 \mu$ (fig. 1, A).

La grande majorité des femelles étudiées présente au niveau de la vulve, une formation ventrale et latéro-ventrale qui ne peut être considérée comme un anneau de copulation, car elle se trouve à l'intérieur du corps. Sur coupes histologiques, cette formation apparaît sous forme de petites nappes ponctuées en rouge après coloration à l'azan. Elles sont situées dans la cavité générale, entre les faisceaux musculaires et dans l'épaisseur de la cuticule (fig. 3).

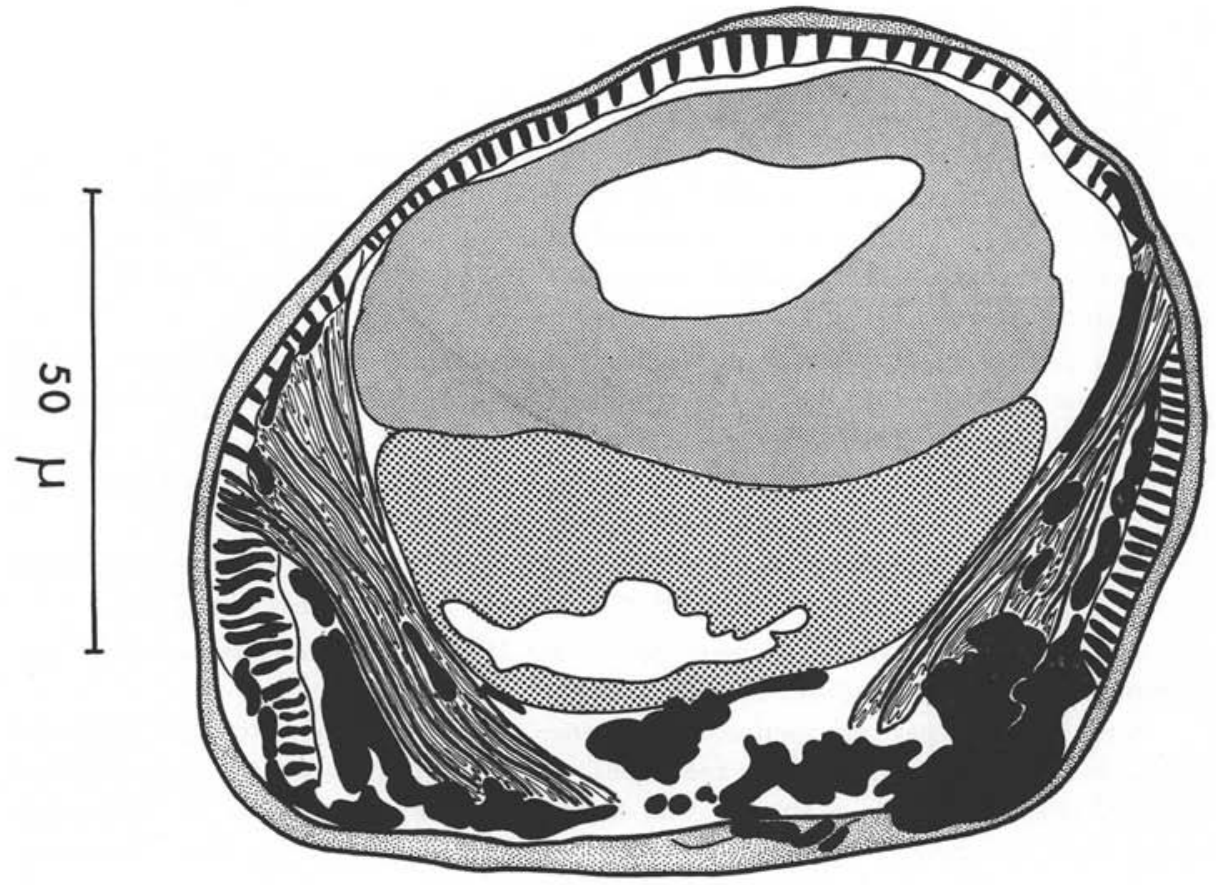

FIG. 3. - Heligmosomoides travassosi Schluz, 1926

†, coupe transversale du corps au niveau de la vulve

\section{Bibliographie}

Durette-Desset (M. C.), 1967. - Evolution des Nématodes Héligmosomes en rapport avec celle de leurs hôtes fondamentaux, les Microtidae. C.R. Acad. Sc. Paris, sér. D, 265, 1500-1503.

Foor (W. E.), Johnson (M. H.) et Beaver (P. C.), 1971. - Morphological changes in the spermatozoa of Dipetalonema viteae in utero. J. Parasit., 57, 1163-1169.

Schulz (R.S.), 1926. - Zur Kenntnis der Helminthenfauna der Nagetiere der Union S.S.R. 1. Subordo Strongylata: 1. Fam. Trichostrongylidae Leiper, 1912. Trudy Gosu darstu. Inst. Ekseper. Vet., 4, 5-32. 